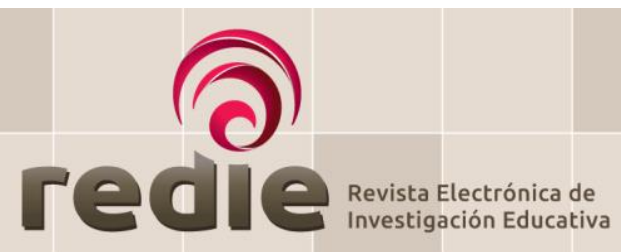

ISSN: $1607-4041$

https://redie.uabc.mx

Vol. 23, 2021/e18

\title{
Diferencia de logro geolocalizado en educación presencial y a distancia en Colombia ${ }^{1}$
}

\section{Geolocated Achievement Differences in Face-to-Face and Distance Education in Colombia}

Nicolás Arias-Velandia (1) https://orcid.org/0000-0002-3798-984X

William Umar Rincón-Báez (2) https://orcid.org/0000-0003-2855-119X

Julián Mauricio Cruz-Pulido (1) https://orcid.org/0000-0002-4407-6184

(1) Institución Universitaria Politécnico Grancolombiano

(2) Corporación Universitaria Minuto de Dios

(Recibido: 19 de diciembre de 2019; Aceptado para su publicación: 5 de mayo de 2020)

Cómo citar: Arias-Velandia, N., Rincón-Báez, W. U. y Cruz-Pulido, J. M. (2021). Diferencia de logro geolocalizado en educación presencial y a distancia en Colombia. Revista Electrónica de Investigación Educativa, 23, e18, 1-22.

https://doi.org/10.24320/redie.2021.23.e18.3711

\section{Resumen}

Los estudiantes exitosos se concentran con frecuencia en las regiones con mayores recursos. Este trabajo indaga la posible incidencia de la ubicación geográfica de la región de los estudiantes sobre su logro, evaluado externamente en programas universitarios de pregrado (presenciales, virtuales y a distancia) de ciencias económicas en instituciones de educación superior en Colombia. Se realizó un análisis mediante modelos georreferenciados y geolocalizados utilizando resultados de evaluación de los estudiantes del sistema FTP-Instituto Colombiano para la Evaluación de la Educación y de la División Político-Administrativa. Se hallaron altos logros en modalidad presencial en regiones centrales como Bogotá y Antioquia, pero también en regiones apartadas en modalidad a distancia tradicional, a pesar de que la ubicación no mostró tendencias estadísticamente significativas en el Modelo de Autocorrelación Espacial I de Moran. Se discuten las tendencias encontradas y los posibles límites de este modelo.

Palabras clave: rendimiento del estudiante, enseñanza superior, aprendizaje en línea, educación a distancia, desequilibrio regional

\section{Abstract}

Successful students are often concentrated in wealthier regions. This paper examines the possible impact of students' geographical location on their achievement, evaluated externally in bachelor's degree programs in economics (taught face-to-face or via traditional or virtual distance learning) in higher education institutions in Colombia. An analysis was conducted using georeferenced and geolocated models, based on students' assessment results from the Colombian Institute for Educational Assessment's (ICFES) FTP system and the Political and Administrative Division (DIVIPOLA) system. High achievement was recorded in face-to-face learning in central regions like Bogotá and Antioquia, but also in more remote regions in traditional distance learning, despite the fact that location did not exhibit statistically significant trends in the spatial autocorrelation measure Moran's I. Lastly, we discuss the trends found and the possible limitations of this model.

Keywords: student achievement, higher education, e-learning, distance education, regional disparities

\footnotetext{
${ }^{1}$ Este trabajo forma parte de la investigación Logro educativo en estudiantes de ciencias económicas en educación superior presencial, distancia tradicional y virtual, financiada por el Instituto Colombiano para la Evaluación de la Educación (ICFES) en la convocatoria de grupos de investigación 2015.
} 


\section{Introducción}

El logro educativo es el aprendizaje que el estudiante alcanza en su participación en un sistema educativo, evidenciado con resultados en evaluaciones externas (Bravo et al., 2017). Al Examen Saber Pro concurren obligatoriamente estudiantes que cursaron $75 \%$ o más de los créditos de los programas de educación superior de pregrado universitario en Colombia (Decreto 3963 de 2009), y pertenece a los exámenes Saber del Instituto Colombiano para la Evaluación de la Educación (ICFES). Evalúa competencias genéricas de comunicación escrita, lectura crítica, razonamiento cuantitativo, inglés y competencias ciudadanas, y competencias específicas según el grupo de referencia de cada programa.

En Colombia existían tres metodologías de programas en educación superior: presencial, con profesores en locaciones de las instituciones en horarios y tiempos específicos; distancia tradicional, con estudiantes siguiendo contenidos por su cuenta con apoyo de TIC en menos del 75\% de instrucción, complementada con encuentros periódicos de estudiantes y profesores en instalaciones de la institución educativa; y distancia virtual, con actividades de estudio personal de los alumnos e interacciones entre ellos y los docentes completamente en línea (Decreto 1295 de 2010).

Las oportunidades de desarrollo en Colombia son más frecuentes en grandes ciudades y en lugares con más recursos, mayor dinámica económica y de comunicaciones, consolidadas en el desarrollo histórico de sus regiones (Colmenares, 1987; Fujita et al., 2001; Romero, 1969/2001). Allí, metodologías de educación a distancia responden a los retos de llevar educación superior a regiones apartadas o alejadas del gran movimiento socioeconómico y cultural, y al de impartir dicha educación con calidad a poblaciones vulnerables, personas en zonas rurales, vinculadas laboralmente o con trabajo en horarios extensos (Lago et al., 2004; Padilla, 2004; Rivera, 2004; Vianney et al., 2004).

Por eso se estudia el logro educativo geolocalizado en Colombia con modelos de estadística espacial (Clark, 1979). Los estudiantes tienden a conglomerarse espacialmente en áreas en las cuales se ubican las instituciones de educación superior, y el prestigio de las instituciones se relaciona también con su cercanía a áreas geográficas con mayor dinámica económica (Aktas et al., 2017; Asmar y Gómez, 2015; Coric y Aleksic-Maslac, 2010; Derbyshire et al., 2014; Ferreira y Cardoso, 2010; Ganapathy, 2015; Gibbons y Vignoles, 2012; Hamnett y Butler, 2011; Helbig et al., 2017; Lokuge, 2010; Mora, 2015, 2016; Mora y Múnera, 2019; Smith et al., 2002).

La distancia del lugar de residencia del estudiante a las instituciones de educación superior se relaciona con el tipo de opciones de carrera en un área geográfica cercana y a los costos de transporte y alojamiento asociados a esta distancia (Aktas et al., 2017; Coric y Aleksic-Maslac, 2010; Cvecic et al., 2019; Derbyshire et al., 2014; Ferreira y Cardoso, 2010; Gibbons y Vignoles, 2012; Hamnett y Butler, 2011; Hanewicz, 2009; Helbig et al., 2017; Ling, 2017; Ma et al., 2017; Smith et al., 2002; Verdis et al., 2019).

En este contexto, los programas de estudios a distancia parecen tener mayor representación de estudiantes de diferentes procedencias geográficas, relacionadas con el mayor acceso que dan a estudiantes de minorías étnicas y de grupos socialmente desfavorecidos (Aktas et al., 2017; Asmar y Gómez, 2015; Coric y Aleksic-Maslac, 2010; Ferreira y Cardoso, 2010; Gibbons y Vignoles, 2012; Hamnett y Butler, 2011; Helbig et al., 2017; Lokuge, 2010; Mora, 2015, 2016).

El trabajo de programas e instituciones de educación superior, apoyado en e-learning y trabajo en línea, se ha presentado como estrategia idónea para llegar a una población más grande de estudiantes, superando barreras geográficas (Coric y Aleksic-Maslac, 2010; Ferreira y Cardoso, 2010; Ganapathy, 2015; Lokuge, 2010; Luo et al., 2014). Asimismo, en el desempeño de los estudiantes a distancia pesan más variables como el rendimiento académico en estudios previos y la edad del estudiante, y menos la distancia del lugar de residencia a la institución (Ganapathy, 2015).

Estas consideraciones tienen implicaciones metodológicas: son importantes las áreas geográficas que se toman como unidad de análisis (Haley, 2017; Smith et al., 2002). La ubicación de la institución del estudiante es crucial para determinar aspectos administrativos para su atención presencial o a distancia (Coric y Aleksic-Maslac, 2010; Derbyshire et al., 2014; Gibbons y Vignoles, 2012; Haley, 2017; Hanewicz, 2009). En 
programas en línea de instituciones de educación superior en México, un criterio para evaluar sus condiciones institucionales es la especificidad de los servicios al estudiante, considerando su tiempo y lugar de residencia (Luna et al., 2018).

Diferentes investigaciones muestran que el logro educativo de los estudiantes colombianos en diferentes niveles educativos cambia en función del espacio geográfico del país en el cual realizan sus estudios (AriasVelandia et al., 2018; Asmar y Gómez, 2015; Celis et al., 2012; Mora, 2015, 2016; Rincón-Báez y AriasVelandia, 2017). Por otra parte, también se ha evidenciado que el logro educativo en niveles educativos previos tiene incidencia en el logro de los estudiantes en educación superior. En este sentido, hay grandes diferencias en dicho logro entre estudiantes de regiones centrales y de grandes ciudades colombianas en contraste con los de otras zonas del país (Asmar y Gómez, 2015; Celis et al., 2012; Mora, 2015, 2016; Restrepo, 2005; Rodríguez et al., 2014).

De 2002 a 2017 buena parte de la cobertura de la educación superior en Colombia creció en las grandes ciudades, especialmente en Bogotá. En los programas virtuales esta cobertura aumentó un 13\% en 2011 y en 2017 ese aumento llegó al 98.9\% (Arias-Velandia et al., 2018; Rincón-Báez et al., 2018) (Centro Nacional de Consultoría, 2017), y pasó de tener un 0.6\% a un 5.6\% del total de estudiantes de educación superior, mientras que los programas a distancia tradicional pasaron del $10.3 \%$ al $12 \%$ de esa cobertura en el mismo período (Sistema Nacional de Información de la Educación Superior [SNIES], 2017).

En consecuencia, es posible que los estudiantes de diferentes zonas del país no cuenten con conocimientos y competencias similares al iniciar programas de educación superior de pregrado universitario (AriasVelandia et al., 2018). La organización tradicional de la educación presencial tiende a agrupar o concentrar estudiantes de regiones centrales y de otras regiones que migran hacia dichas regiones centrales, pero en el caso de la educación a distancia tradicional o virtual, dichos desplazamientos dejan de ser necesarios. Esto quitaría peso a los factores de distancia, pero también hay diferencias importantes de los logros de aprendizaje al finalizar la educación básica y media entre los estudiantes de diferentes regiones.

De todo este conjunto de estudios se concluye la necesidad de caracterizar el logro de los estudiantes en función de variables geográficas o del lugar de residencia del estudiante (Montoya, 2013). Este estudio se pregunta entonces: ¿De qué manera los análisis de georreferenciación o geolocalización nos muestran tendencias particulares de la educación a distancia y la educación virtual, en comparación con programas de educación presencial del área de conocimiento Economía, Administración, Contaduría y afines en instituciones de educación superior de Colombia?

\section{Método}

Este estudio muestra los resultados de la evaluación de genéricas del examen Saber Pro, comparándolos en diferentes departamentos de la División Político-Administrativa (DIVIPOLA) de Colombia², en relación con metodologías presencial, distancia tradicional y distancia virtual, de programas de Ciencias Económicas. Estos abarcan el $27 \%$ de todos los estudiantes de programas de educación superior universitaria en Colombia (Arias-Velandia et al., 2018) con algunos de los programas más antiguos en metodologías no presenciales (El Espectador, 2014; Ministerio de Educación Nacional, 2014; SNIES, 2017).

En la investigación, de tipo retrospectivo con alcance correlacional (Hernández et al., 2014), se utilizaron las bases de datos del Examen Saber Pro de los años 2012 y 2014, extraídas del sistema de información que sirve para acceder a bases de datos del ICFES a través de un protocolo FTP (File Transfer Protocol). Estas bases de datos (6 en total) se acoplan con las del SNIES del Ministerio de Educación Nacional de Colombia.

Con las bases de datos del sistema FTP-ICFES Saber Pro (ICFES, 2019) se filtran los programas del área de Economía, Administración, Contaduría y afines, y de instituciones que cuentan con programas de esta área en modalidad presencial y a distancia (tradicional o virtual), por medio de su conexión con la base de

\footnotetext{
2 División Política Administrativa (DIVIPOLA) es un sistema de información geográfica vía web que permite la consulta en el territorio nacional de la División Político-Administrativa. Constituye información fundamental para el diseño de políticas, provisión de servicios públicos, así como la definición de criterios para la asignación de recursos por parte del gobierno central" (Departamento Administrativo Nacional de Estadística, 2019).
} 
instituciones del SNIES (Ministerio de Educación Nacional, 2020). Participaron 20 instituciones de educación superior colombianas, con programas de ciencias económicas, empresariales, administrativas y afines, que tenían ofertas equivalentes en programas presenciales y a distancia (virtual o tradicional) y que contaban con egresados que presentaron el Examen Saber Pro.

Adicionalmente, se retiraron de las bases de datos los registros de estudiantes que: 1) tienen valores de cero en los puntajes de al menos una de las pruebas del examen Saber Pro, debido a que no es claro si el estudiante estuvo o no presentando esta prueba (dado que un patrón aleatorio de respuestas tendría al menos una probabilidad de 0.25 , que es mayor que la puntuación cero) y sus datos generan valores que perturban su conjunto total, o 2) manifiestan tener limitaciones físicas y cognitivas, ya que no serían comparables con el conjunto de datos y son muy pocos (menos de 20 participantes), por lo cual su información no permite extraer inferencias estadísticas con este grupo. Con esta información se generó la Base Maestra que se utilizó en el análisis posterior; la base incluyó 90 campos de puntajes de estudiantes y de variables sociales, económicas, de estudios previos y de acceso a medios de cada uno de ellos, y 10388 registros correspondientes al mismo número de estudiantes que presentaron el Examen Saber Pro en 2012, 2013 y 2014 en el área de conocimiento de Economía, Administración, Contaduría y afines en la base de datos de SNIES.

El $85.9 \%$ eran de metodología presencial, $12.7 \%$ de distancia tradicional y $1.4 \%$ de virtual. Sobre la base de datos maestra se realizó el análisis de georreferenciación de la misma base de datos, usando aplicaciones en el framework shiny de $\mathrm{R}^{3}$ (R Development Core Team, 2008).

\subsection{Análisis de información y análisis estadístico georreferenciado}

La metodología del estudio busca situar espacialmente a los estudiantes y programas académicos correspondientes a Economía, Administración, Contaduría y afines a nivel nacional, para cada una de las modalidades: presencial, distancia tradicional y distancia virtual. Adicionalmente, se evalúa la hipótesis de correlación espacial, que permite evaluar la existencia de un mayor (o menor) rendimiento educativo en unas áreas geográficas determinadas del país respecto a otras (Schabenberger y Gotway, 2017).

El análisis georreferenciado se divide en dos procedimientos: 1) modelo georreferenciado con modelo estadístico de áreas y prueba de autocorrelación (análisis de áreas), que indaga a través de métodos de estadística espacial la relación entre el logro académico y la localización de los estudiantes (Clark, 1979); 2) análisis comparativo con resultados geoespaciales descriptivos (análisis comparativo).

Análisis de áreas. Se realiza análisis de áreas motivado por georreferenciación de los datos en regiones definidas. El primer paso para formularlo y ajustarlo es establecer una matriz de vecindades (Massey y Denton, 1988), o arreglo binario que tiene valor uno (1) cuando dos áreas son vecinas o comparten frontera, y cero (0) cuando no lo son. Esta matriz de vecindades constituye el insumo principal para establecer la posible existencia de correlación espacial. Este procedimiento estadístico espacial examina la presencia de un fenómeno en una región o zona geográfica, en relación con su presencia o con su probable presencia en zonas geográficas vecinas (Moran, 1948).

Análisis comparativo. El análisis comparativo es realizado a nivel descriptivo. Se realiza dado que el examen Saber Pro tiene cobertura nacional y es requisito obligatorio para la obtención del título de grado de un programa de pregrado, lo cual garantiza que tenga cobertura poblacional. Este análisis permite establecer las tendencias regionales en cobertura y desempeño de las tres metodologías: presencial, distancia tradicional y distancia virtual.

${ }^{3}$ En https://goo.gl/ZN74mx y https://goo.gl/ODylPQ 


\section{Resultados}

\subsection{Análisis de georreferenciación}

En los programas del área de Economía, Administración, Contaduría y afines hay más estudiantes de Departamentos de la costa sobre el mar Caribe, con costa sobre el océano Pacífico y de la zona andina montañosa al interior de Colombia. A pesar del tamaño muestral de 10388 estudiantes, la mayoría de los municipios en el territorio nacional no se encuentra representado en la muestra (Figura 1).

Figura 1. Cantidad de estudiantes evaluados por municipio

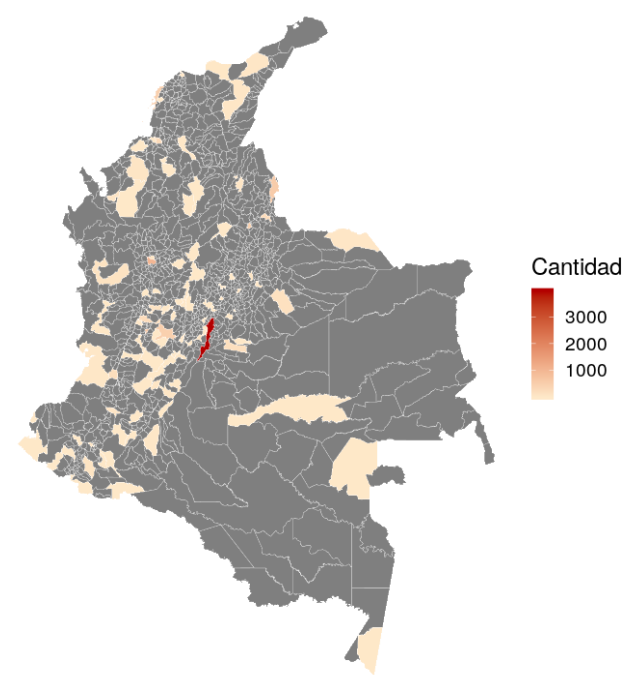

Fuente: Resultados del examen Saber Pro 2012-2014.

La cantidad de estudiantes evaluados por Departamentos en los programas de este estudio disminuye el nivel de desagregación territorial de la muestra. Así, 2 de los 32 departamentos registrados en la DIVIPOLA (Figura 2) carecen de datos para este estudio.

Figura 2. Cantidad de estudiantes evaluados por departamento

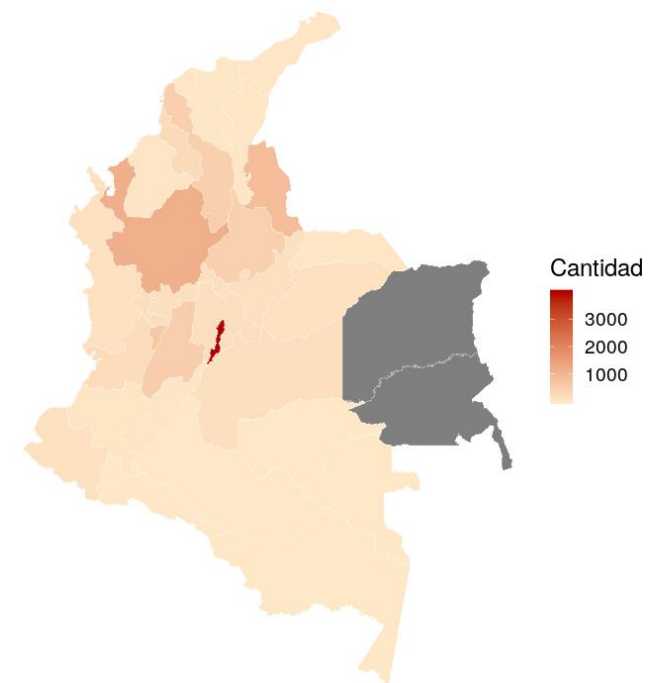

Fuente: Resultados del examen Saber Pro 2012-2014. 
En concordancia con esta cobertura, en la Figura 3 se muestra el puntaje global promedio por municipio. La dispersión geográfica de los municipios representados en la muestra no permite una interpretación intuitiva. Debido a ello, se presentaron los datos agregados a nivel departamental, permitiendo una mejor aproximación en los programas del área (Figura 4). Aquí es posible observar desempeños más altos en los Departamentos de Atlántico, Bolívar (costa del Caribe) Santander, Bogotá (zona central de los Andes); y otros con puntajes promedio destacados como Caldas, Tolima (zona central de los Andes), Meta (Llanura Oriental) y Vaupés (zona Amazónica) (ver Tabla 1).

Figura 3. Puntaje global promedio por municipio

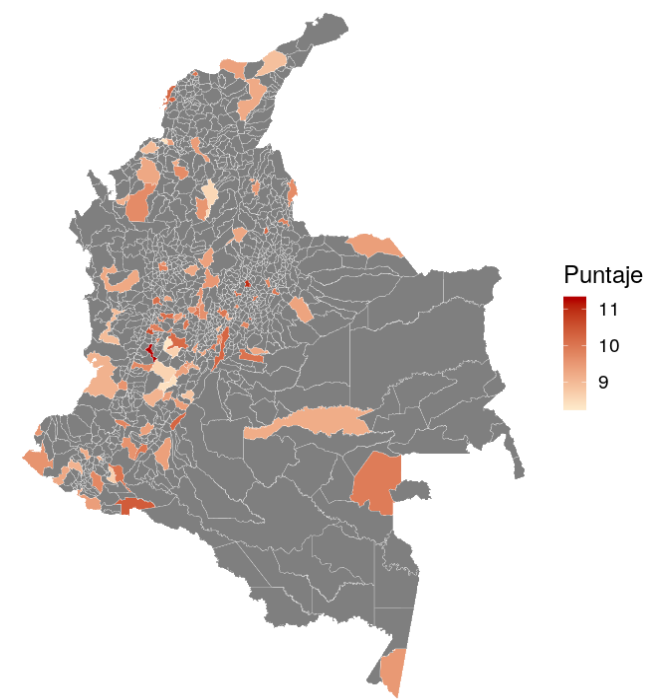

Fuente: Resultados del Examen Saber Pro 2012-2014, ICFES.

Figura 4. Puntaje global promedio por departamento

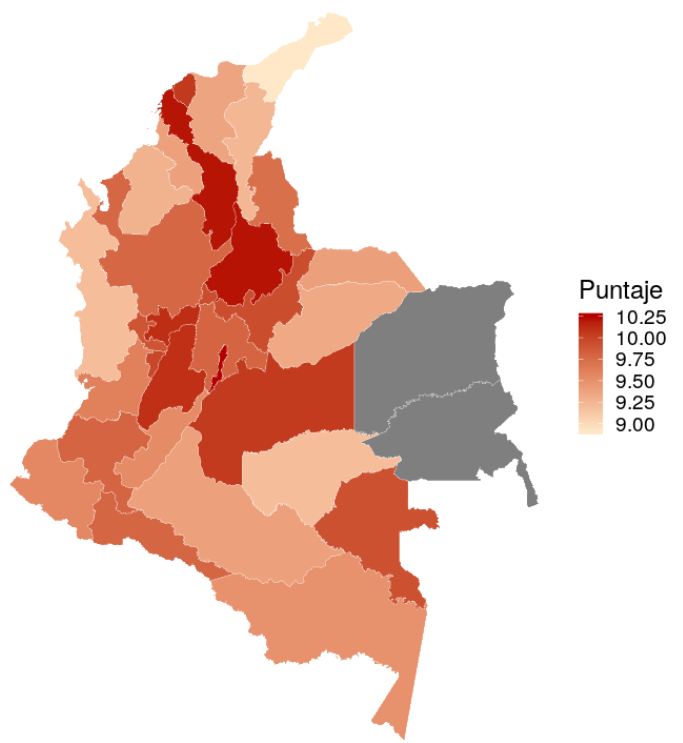

Fuente: Resultados del Examen Saber Рro 2012-2014, ICFES. 


\subsection{Matriz de vecindades}

A continuación se muestra la matriz de vecindades de los municipios de Colombia (Figura 5).

Figura 5. Matriz de vecindades usando la totalidad de los municipios de Colombia

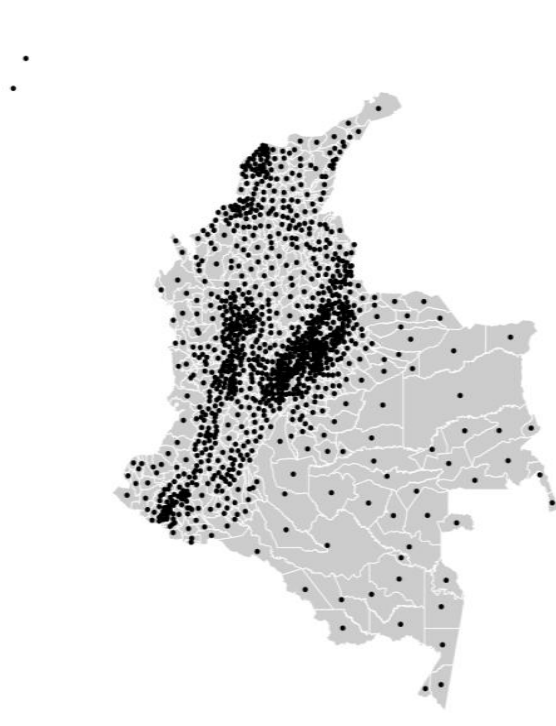

Fuente: DIVIPOLA.

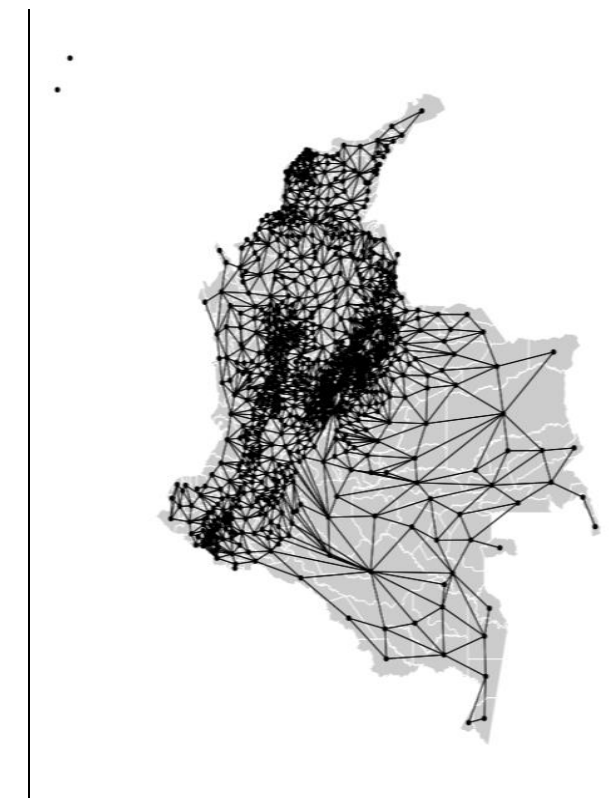

La DIVIPOLA comprende 1101 municipios, la matriz de vecindades ilustrada mide 606651 posibles vecindades. Por otro lado, al calcular la matriz de vecindades con los datos de este estudio, se muestra que el panorama es distinto (Figura 6).

Figura 6. Matriz de vecindades con municipios pertenecientes a la muestra
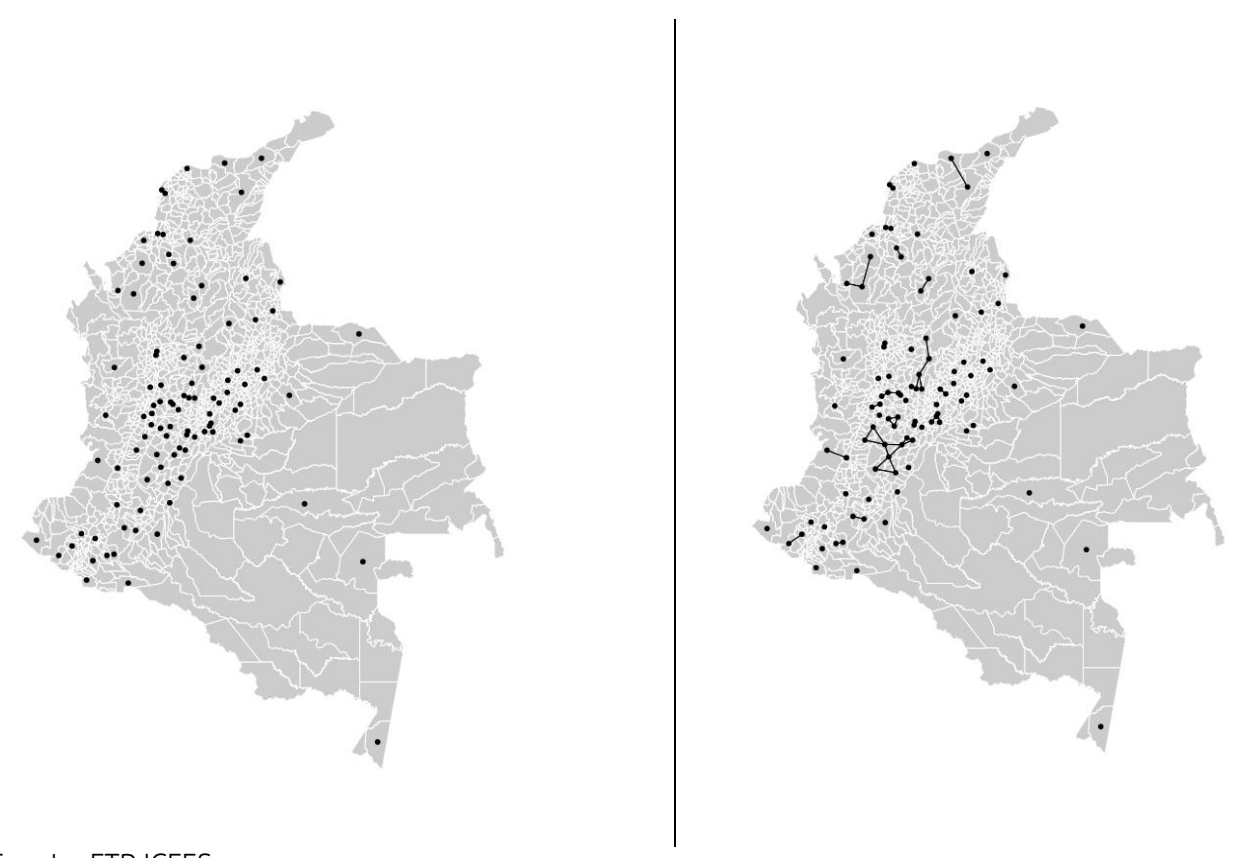

Fuente: FTP-ICFES. 
El contraste es notorio ya que debido al nivel de desagregación, los municipios vecinos incluidos en la muestra son muy pocos. Por lo tanto, no es posible realizar un modelo de áreas usando la información por municipios. En contraste, la información a nivel departamental se encuentra más completa. La matriz de vecindades da cuenta de ello (Figura 7).

Figura 7. Matriz de vecindades de los departamentos de la muestra
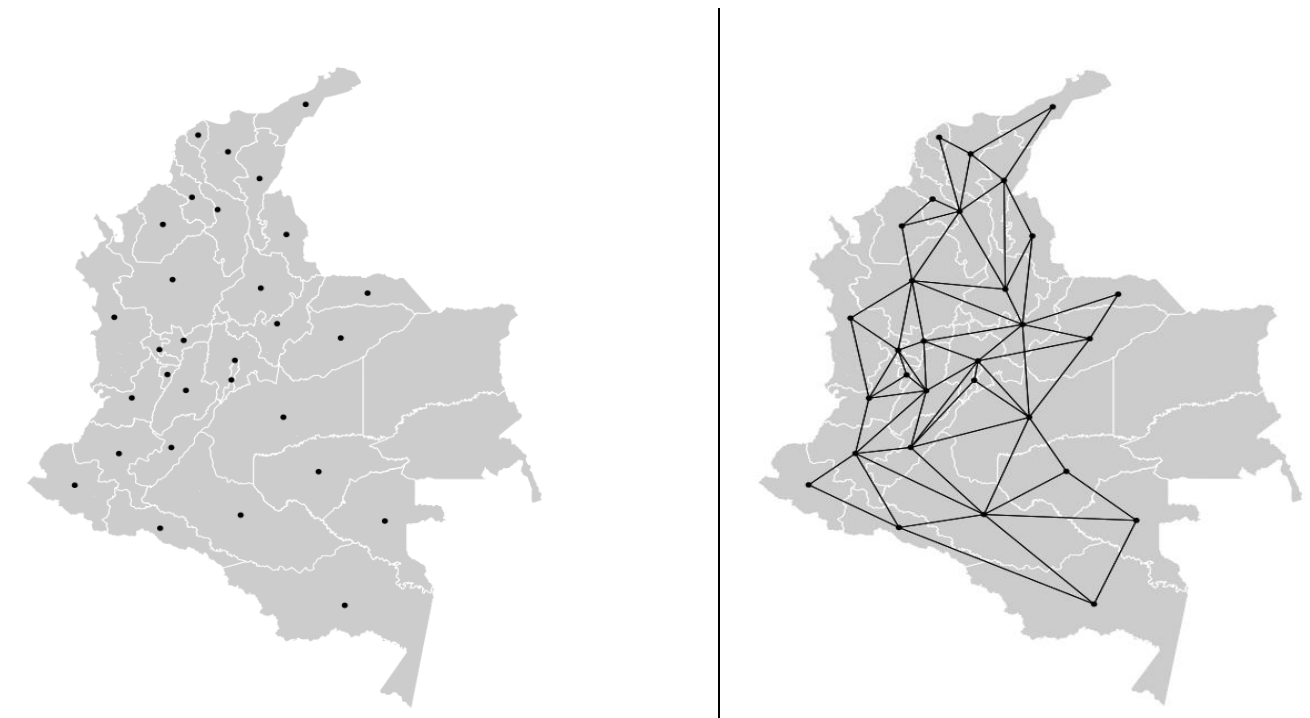

Fuente: FTP-ICFES.

\subsection{Pruebas de autocorrelación}

La ausencia de municipios vecinos restringe el análisis correlacional sólo a los departamentos, realizado con correlación espacial de Moran (1948) (Tabla 1). La prueba no detectó evidencia estadísticamente significativa de esta autocorrelación, por lo que no es posible afirmar que el rendimiento académico de los estudiantes de los programas seleccionados se deba a su ubicación regional.

Tabla 1. Salida de la prueba de autocorrelación espacial de Moran

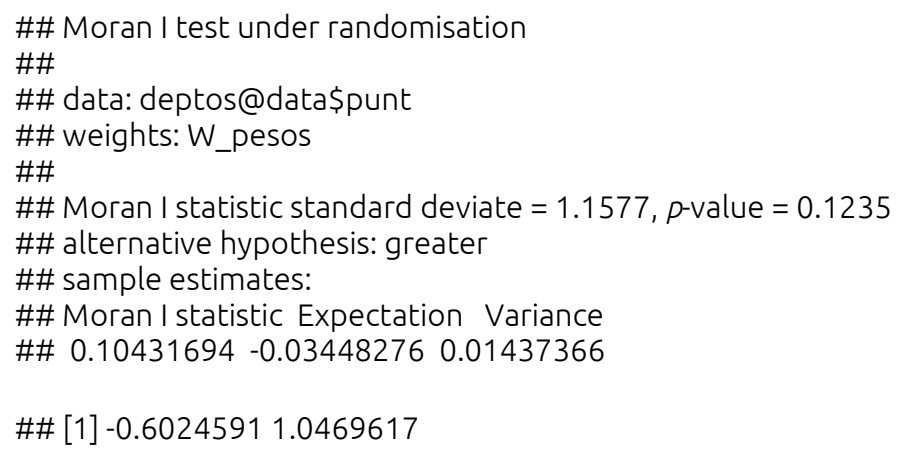

Fuente: FTP-ICFES. 
Este resultado también es afectado por el tamaño muestral: con pocas áreas (30 Departamentos) es difícil establecer niveles de significancia aceptables. Este análisis en el nivel de municipios podría tener mejores resultados, pero la poca cobertura de los programas seleccionados genera una fuerte dispersión y poca conectividad entre las áreas, como se observa en mapas previos.

La prueba de Moran no detecta evidencia estadísticamente significativa de correlación espacial. En consecuencia, no resulta acertado ajustar un modelo estadístico con carácter georreferenciado. Se muestran entonces los estadísticos descriptivos señalados anteriormente, agrupándolos según el método del programa: presencial, distancia tradicional y distancia virtual. 


\subsection{Estadísticos descriptivos espaciales comparativos de metodología presencial, distancia tradicional y virtual}

Es posible observar que la educación a distancia cobija territorios que no resultan accesibles a otros tipos de educación (Tabla 2; figuras 8 y 9).

Tabla 2. Número de estudiantes y sus puntajes promedio en Saber Pro por Departamentos en la División Político-Administrativa de Colombia

\begin{tabular}{|c|c|c|c|c|c|c|c|c|c|}
\hline ID & Departamento & Estudiantes & $\begin{array}{l}\text { Puntaje } \\
\text { Promedio }\end{array}$ & $\begin{array}{l}\text { Núm. } \\
\text { Estudiantes } \\
\text { Presencial }\end{array}$ & $\begin{array}{c}\text { Núm. } \\
\text { Estudiantes } \\
\text { Distancia } \\
\text { Tradicional }\end{array}$ & $\begin{array}{l}\text { Núm. } \\
\text { Estudiantes } \\
\text { Virtual }\end{array}$ & $\begin{array}{l}\text { Puntaje } \\
\text { Promedio } \\
\text { Presencial }\end{array}$ & $\begin{array}{c}\text { Puntaje } \\
\text { Promedio } \\
\text { Distancia } \\
\text { Tradicional }\end{array}$ & $\begin{array}{c}\text { Puntaje } \\
\text { Promedio } \\
\text { Virtual }\end{array}$ \\
\hline 5 & Antioquia & 1135 & 9.78 & 309 & 826 & 0 & 10.24 & 9.61 & $\mathrm{~N} / \mathrm{A}$ \\
\hline 8 & Atlántico & 27 & 10.06 & 0 & 27 & 0 & $\mathrm{~N} / \mathrm{A}$ & 10.06 & $\mathrm{~N} / \mathrm{A}$ \\
\hline 11 & Bogotá & 3971 & 10.25 & 2866 & 877 & 228 & 10.40 & 9.78 & 10.23 \\
\hline 13 & Bolívar & 512 & 10.21 & 335 & 177 & 0 & 10.54 & 9.59 & $\mathrm{~N} / \mathrm{A}$ \\
\hline 15 & Boyacá & 171 & 9.95 & 40 & 131 & 0 & 10.15 & 9.89 & $\mathrm{~N} / \mathrm{A}$ \\
\hline 17 & Caldas & 157 & 10.12 & 35 & 100 & 22 & 11.07 & 9.81 & 9.99 \\
\hline 18 & Caquetá & 12 & 9.40 & 0 & 12 & 0 & $\mathrm{~N} / \mathrm{A}$ & 9.40 & $\mathrm{~N} / \mathrm{A}$ \\
\hline 19 & Cauca & 52 & 9.80 & 0 & 52 & 0 & $\mathrm{~N} / \mathrm{A}$ & 9.80 & $\mathrm{~N} / \mathrm{A}$ \\
\hline 20 & Cesar & 36 & 9.25 & 0 & 36 & 0 & $\mathrm{~N} / \mathrm{A}$ & 9.25 & $\mathrm{~N} / \mathrm{A}$ \\
\hline 23 & Córdoba & 64 & 9.28 & 21 & 43 & 0 & 9.23 & 9.30 & $\mathrm{~N} / \mathrm{A}$ \\
\hline 25 & Cundinamarca & 185 & 9.80 & 1 & 184 & 0 & 9.91 & 9.80 & $\mathrm{~N} / \mathrm{A}$ \\
\hline 27 & Chocó & 138 & 9.20 & 104 & 34 & 0 & 9.25 & 9.07 & $\mathrm{~N} / \mathrm{A}$ \\
\hline 41 & Huila & 69 & 9.54 & 0 & 69 & 0 & $\mathrm{~N} / \mathrm{A}$ & 9.54 & $\mathrm{~N} / \mathrm{A}$ \\
\hline 44 & Guajira & 38 & 8.91 & 0 & 38 & 0 & $\mathrm{~N} / \mathrm{A}$ & 8.91 & $\mathrm{~N} / \mathrm{A}$ \\
\hline 47 & Magdalena & 30 & 9.37 & 0 & 30 & 0 & $\mathrm{~N} / \mathrm{A}$ & 9.37 & $\mathrm{~N} / \mathrm{A}$ \\
\hline 50 & Meta & 191 & 10.05 & 133 & 58 & 0 & 10.14 & 9.85 & $\mathrm{~N} / \mathrm{A}$ \\
\hline 52 & Nariño & 137 & 9.57 & 0 & 137 & 0 & $\mathrm{~N} / \mathrm{A}$ & 9.57 & $\mathrm{~N} / \mathrm{A}$ \\
\hline 54 & Norte de Santander & 865 & 9.73 & 609 & 256 & 0 & 9.79 & 9.59 & $\mathrm{~N} / \mathrm{A}$ \\
\hline 63 & Quindío & 626 & 9.99 & 201 & 425 & 0 & 10.43 & 9.78 & $\mathrm{~N} / \mathrm{A}$ \\
\hline 66 & Risaralda & 235 & 9.90 & 154 & 81 & 0 & 9.95 & 9.80 & $\mathrm{~N} / \mathrm{A}$ \\
\hline 68 & Santander & 475 & 10.22 & 224 & 251 & 0 & 10.65 & 9.83 & $\mathrm{~N} / \mathrm{A}$ \\
\hline 70 & Sucre & 271 & 9.40 & 121 & 150 & 0 & 9.55 & 9.28 & $\mathrm{~N} / \mathrm{A}$ \\
\hline 73 & Tolima & 531 & 10.11 & 185 & 346 & 0 & 10.95 & 9.65 & $\mathrm{~N} / \mathrm{A}$ \\
\hline 76 & Valle del Cauca & 248 & 9.61 & 0 & 248 & 0 & $N / A$ & 9.61 & $\mathrm{~N} / \mathrm{A}$ \\
\hline 81 & Arauca & 40 & 9.41 & 0 & 40 & 0 & $\mathrm{~N} / \mathrm{A}$ & 9.41 & $\mathrm{~N} / \mathrm{A}$ \\
\hline 85 & Casanare & 140 & 9.34 & 0 & 140 & 0 & $\mathrm{~N} / \mathrm{A}$ & 9.34 & $\mathrm{~N} / \mathrm{A}$ \\
\hline 86 & Putumayo & 13 & 9.80 & 0 & 13 & 0 & $\mathrm{~N} / \mathrm{A}$ & 9.80 & $\mathrm{~N} / \mathrm{A}$ \\
\hline 91 & Amazonas & 5 & 9.50 & 0 & 5 & 0 & $\mathrm{~N} / \mathrm{A}$ & 9.50 & $\mathrm{~N} / \mathrm{A}$ \\
\hline 95 & Guaviare & 8 & 9.21 & 0 & 8 & 0 & $\mathrm{~N} / \mathrm{A}$ & 9.21 & $\mathrm{~N} / \mathrm{A}$ \\
\hline 97 & Vaupés & 6 & 9.93 & 0 & 6 & 0 & $\mathrm{~N} / \mathrm{A}$ & 9.93 & $\mathrm{~N} / \mathrm{A}$ \\
\hline
\end{tabular}


Figura 8. Cantidad de estudiantes por municipio, distancia tradicional

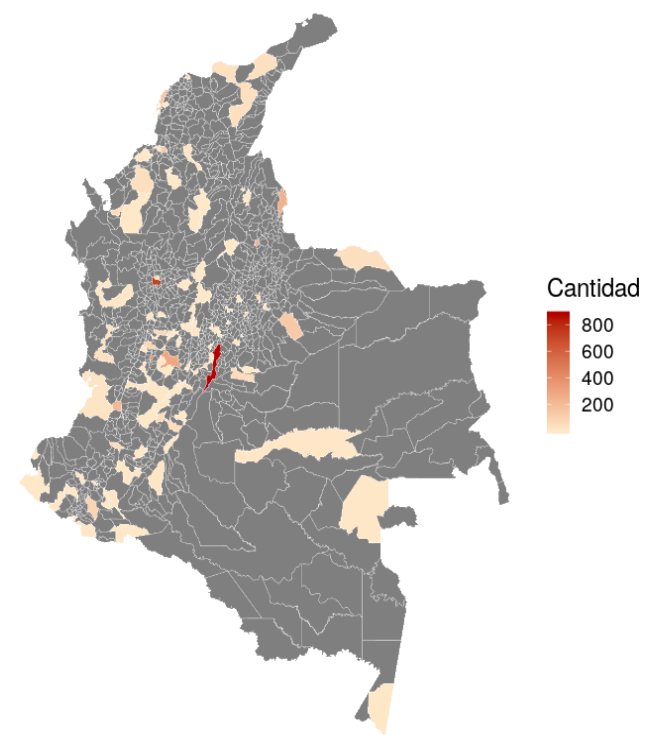

Fuente: Resultados del Examen Saber Pro 2012-2014, ICFES.

Figura 9. Cantidad de estudiantes por departamento, distancia tradicional

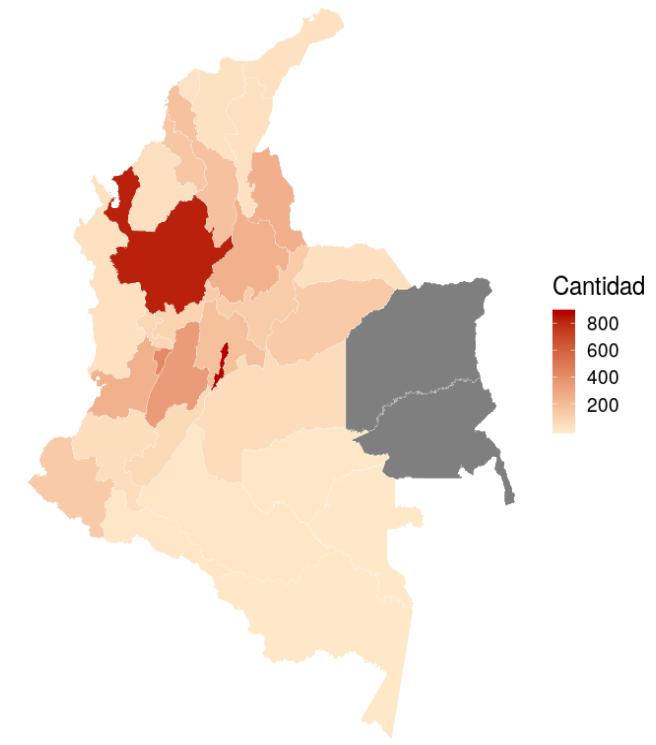

Fuente: Resultados del Examen Saber Pro 2012-2014, ICFES.

Los puntajes de los estudiantes de la metodología a distancia tradicional en los programas de Economía, Administración, Contaduría y afines son más bajos que el puntaje global promedio. Sin embargo, en la metodología a distancia tradicional hay muchos más estudiantes en Bogotá y Antioquia (Figura 10), pero se observan puntajes promedio más altos en regiones y Departamentos como Atlántico (de la Costa del Caribe), Vaupés, Putumayo (ambos de la Selva Amazónica), Meta (de la Llanura Oriental), Boyacá, Caldas, Risaralda, Quindío (de la zona de los Andes) y Cauca (de la zona de los Andes pero volcado hacia la costa del Pacífico) (figuras 10 y 11). 
Figura 10. Puntaje promedio por municipio, distancia tradicional

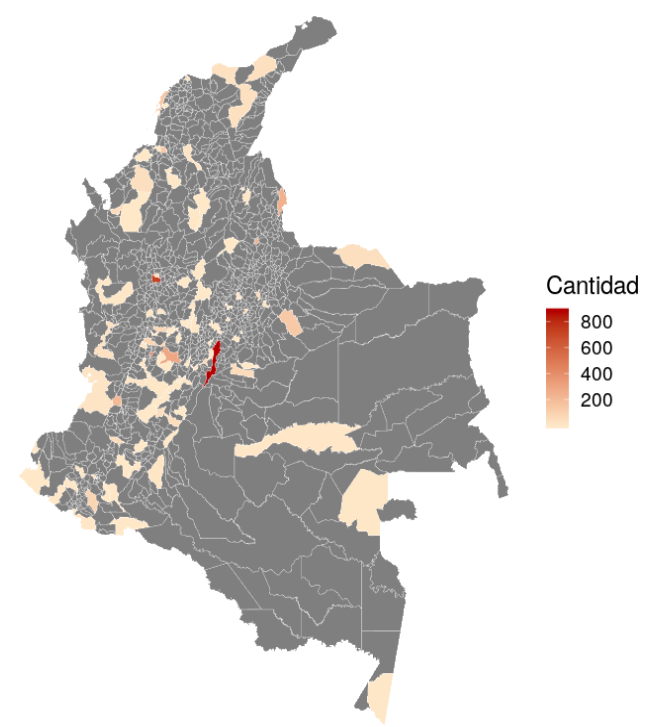

Fuente: FTP-ICFES.

Figura 11. Puntaje promedio por departamento, distancia tradicional

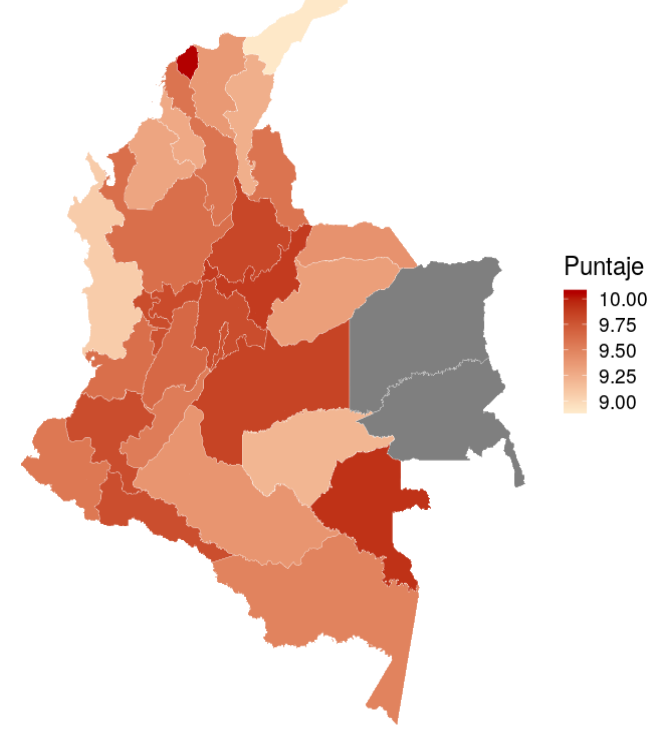

Fuente: FTP-ICFES. 
Los datos de la metodología a distancia virtual en los programas de Economía, Administración, Contaduría y afines, correspondientes al $2.41 \%$ de la muestra, se encuentran localizados en pocos municipios con muy pocos egresados (figuras 12 y 13 ).

Figura 12. Cantidad de estudiantes por municipio, virtual

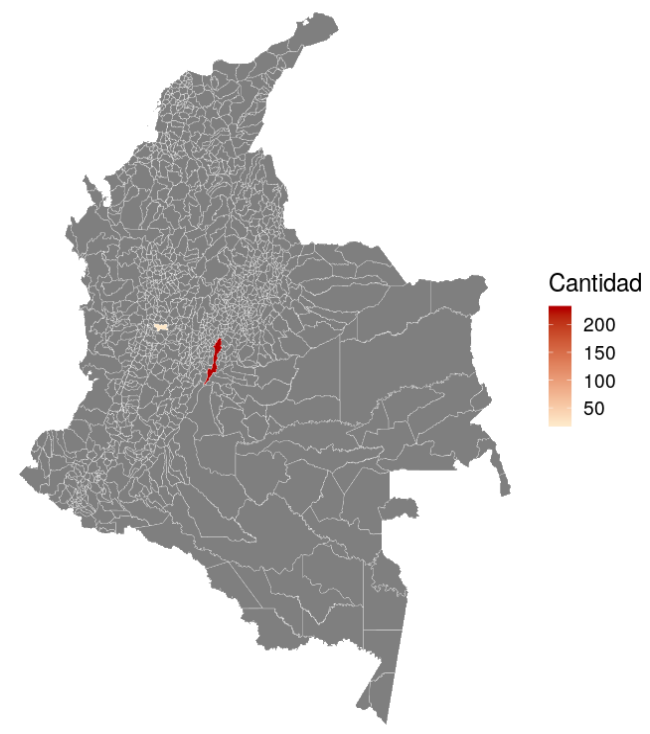

Fuente: FTP-ICFES.

Figura 13. Cantidad de estudiantes por departamento, virtual

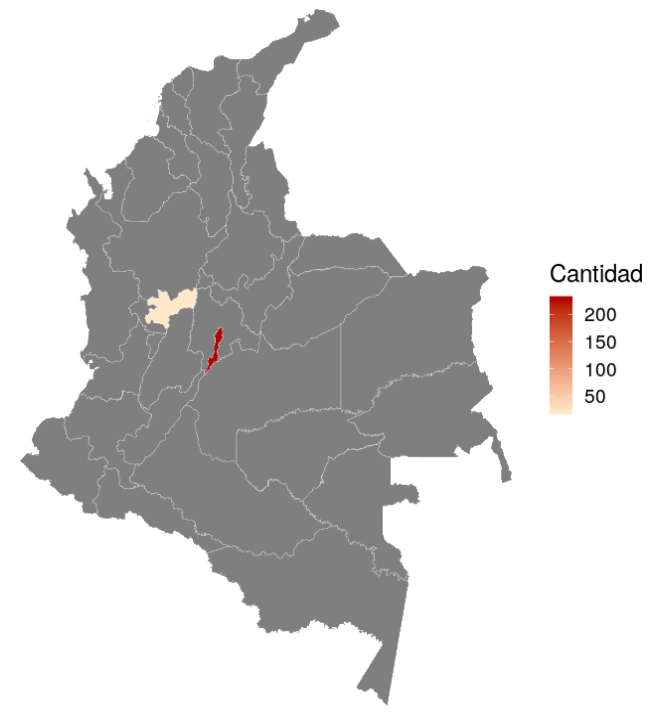

Fuente: FTP-ICFES. 
La implementación de educación virtual en los programas de Economía, Administración, Contaduría y afines entre 2012 y 2014 sólo aporta estudiantes presentando Saber Pro en el departamento de Caldas y en el Distrito Capital (figuras 14 y 15).

Figura 14. Puntaje promedio por municipio, virtual

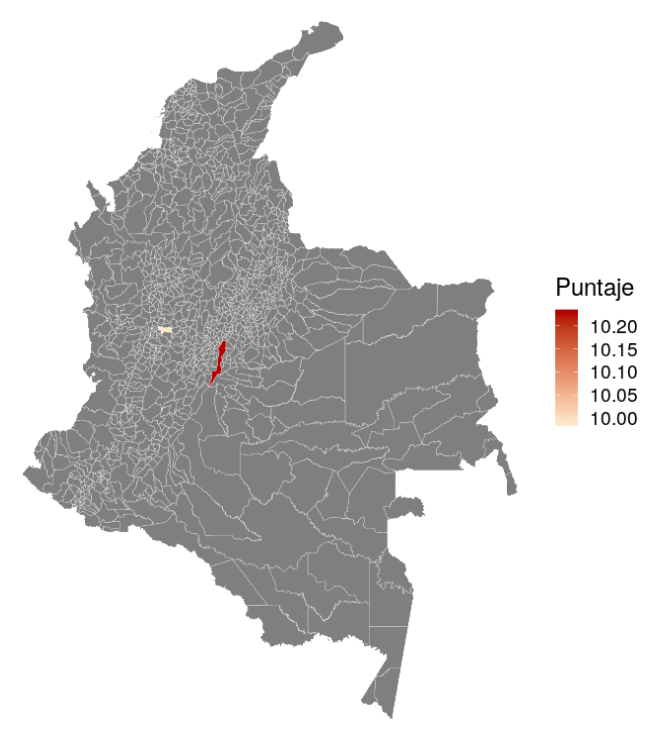

Fuente: FTP-ICFES.

Figura 15. Puntaje promedio por departamento, virtual

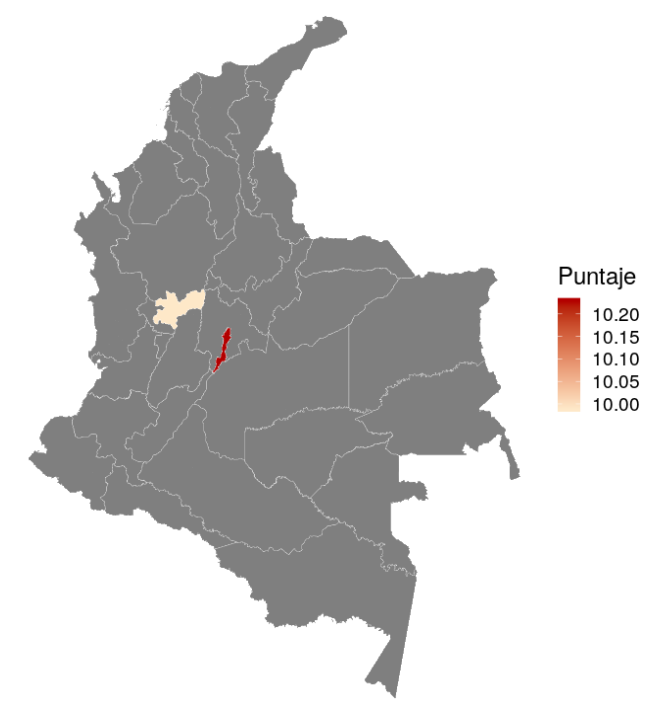

Fuente: FTP-ICFES.

La educación superior en metodología presencial en los programas de Economía, Administración, Contaduría y afines tiene una implementación mayor que la educación en metodología en distancia tradicional por cantidad de estudiantes, pero carece por completo de su cobertura. La metodología presencial en los programas de Ciencias Económicas cobija sólo la zona central del país. ${ }^{4}$ La mayor parte de los estudiantes presenciales de estos programas se ubican en Bogotá, con una menor cantidad en otros

\footnotetext{
${ }^{4}$ El estudio en detalle de los datos agrupados por la metodología del programa revela que en los programas de Economía, Administración, Contaduría y afines son escasas las regiones que poseen los tres tipos de programas. Esto dificulta el cálculo de un modelo estadístico de tipo espacial.
} 
Departamentos centrales (figuras 16 y 17), y los mayores puntajes se encuentran en Bogotá, Caldas y Tolima (todos Departamentos de la zona central de Colombia) (figuras 18 y 19).

Figura 16. Cantidad de estudiantes por municipio, presencial

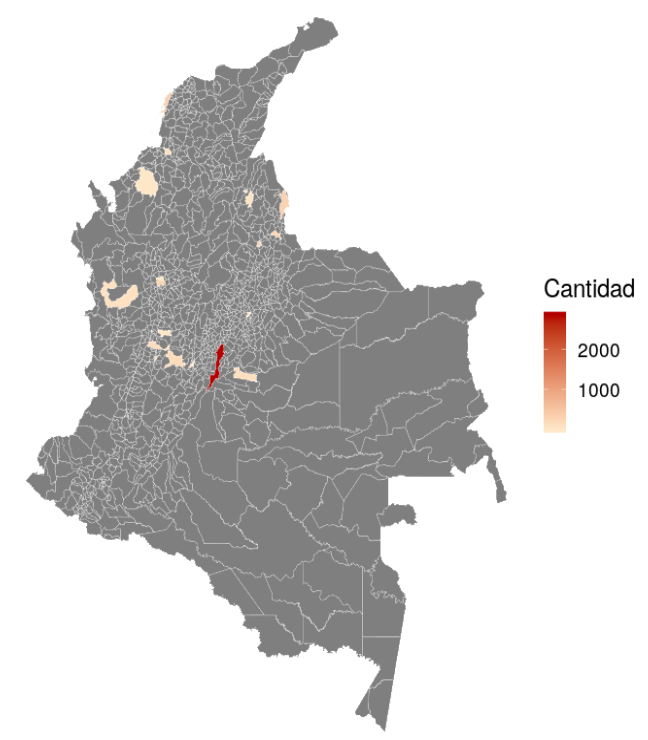

Fuente: FTP-ICFES.

Figura 17. Cantidad de estudiantes por departamento, presencial

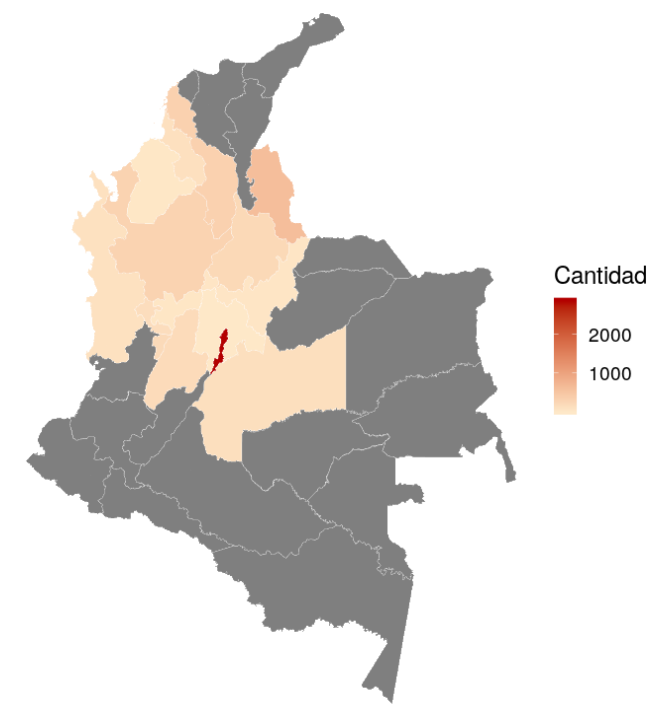

Fuente: FTP-ICFES. 
Figura 18. Puntaje promedio por municipio, presencial

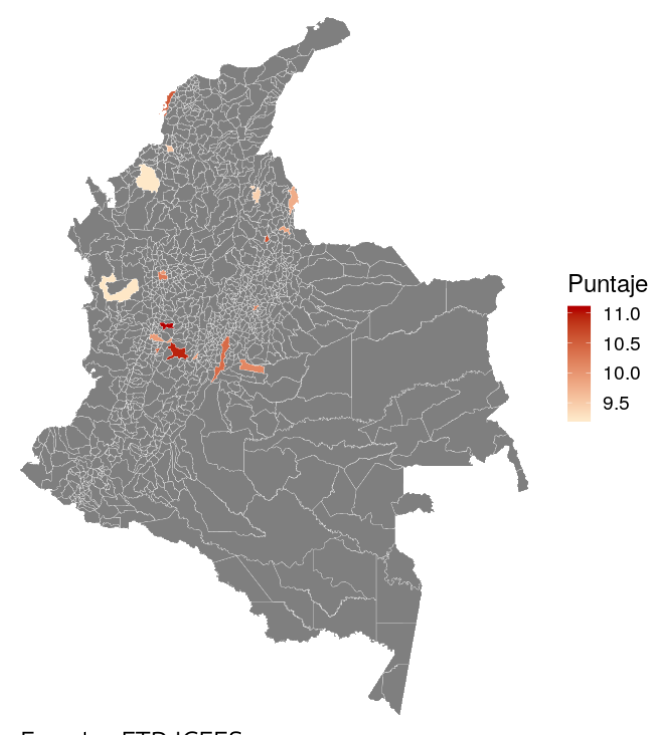

Fuente: FTP-ICFES.

Figura 19. Puntaje promedio por departamento, presencial

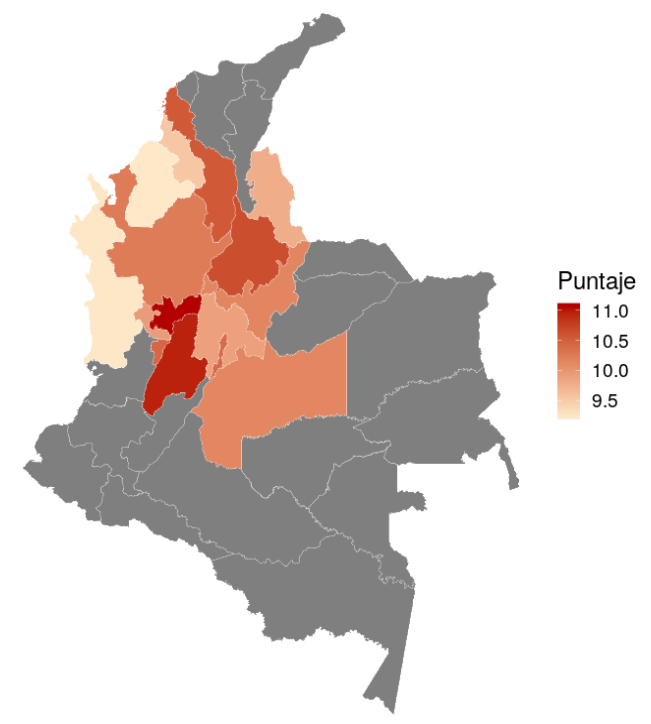

Fuente: FTP-ICFES.

\section{Discusión}

En este documento se presenta un panorama geográfico de la educación superior en el área de Economía, Administración, Contaduría y afines en metodologías presencial, a distancia virtual y a distancia tradicional. La distribución de la educación superior en Colombia no es equitativa. Si bien se ha promovido la cobertura por parte del Ministerio de Educación Nacional (Rincón-Báez et al.., 2018), la misma es apenas del 38\% en 2017 y continúa su tendencia de concentración hacia los grandes núcleos urbanos (Asmar y Gómez, 2015; Mora, 2015, 2016). Es importante el esfuerzo en expandir la cobertura con diferentes estrategias en los últimos años en la educación a distancia tradicional y virtual.

Los resultados muestran que la cobertura en educación superior no se desarrolla necesariamente con calidad en la misma. Existen factores económicos, sociales y de afectación por diferentes tipos de violencia 
armada, que generan inequidad entre las diferentes regiones colombianas. Esto, a su vez, ha llevado a bajos resultados en pruebas estandarizadas censales de rendimiento académico de los estudiantes en zonas apartadas (Arias-Velandia, 2018). Grandes entidades territoriales como Bogotá y Antioquia tienen los mejores puntajes y concentran también la mayor cantidad de instituciones de educación superior (IES). En otras zonas hay menor presencia de comercio y de generadores de empleo, junto con escasez de profesionales especializados o con alto nivel de formación (Arias-Velandia et al., 2018; Asmar y Gómez, 2015; Mora, 2015, 2016).

Entre los hallazgos de este estudio se encontró que la educación a distancia tradicional, si bien evidencia menores logros en sus estudiantes cuando se les compara con los de educación presencial o a distancia virtual, logra tener estudiantes con altos puntajes en el examen Saber Pro en los Departamentos de Atlántico, Meta, Cauca, Putumayo y Vaupés. Esto nos muestra que en el período estudiado, la educación a distancia tradicional tiene estudiantes con altos logros en regiones colombianas que no pertenecen a las regiones centrales, en las que tradicionalmente se encuentran estudiantes con mayores resultados en las evaluaciones del sistema educativo. Esto indica que la educación a distancia tradicional, a pesar de su desventaja comparativa con otras metodologías, muestra evidencias de estar cumpliendo en Colombia el propósito de la UNESCO (2010) Para la educación superior en modalidades no presenciales: llevar la educación superior a poblaciones que habitualmente no tenían acceso a ella (Celis et al., 2012; Guarín et al., 2016; Montoya, 2013).

El hallazgo anterior también tiene otro significado o interpretación: si bien los espacios geográficos colombianos de mayor concentración de recursos y dinámicas económicas y de comunicaciones (Colmenares, 1987; Fujita et al., 2001) suelen agrupar estudiantes con mejores resultados en la evaluación externa de finalización de la educación universitaria de pregrado, otros espacios geográficos ubicados por fuera de dicha concentración presentan varios estudiantes con alto logro en dicha evaluación. Lo anterior muestra, para el caso de Colombia, una confirmación de que la educación a distancia tiene aspectos con diferente peso en el aprendizaje de sus estudiantes, especialmente en lo relacionado con su geolocalización (Coric y Aleksic-Maslac, 2010; Ferreira y Cardoso, 2010; Ganapathy, 2015; Lokuge, 2010; Luo et al., 2014), por lo cual puede ser una fuente de estrategias de cierre de brechas educativas entre diferentes regiones de Colombia (Arias-Velandia et al., 2018; Rincón-Báez et al., 2018). En hallazgos recientes, esto parece ser cierto también en el caso de estudiantes de educación virtual cuando realizan trabajos de iniciación en la investigación en sus lugares de origen como parte de su formación (Cortés et al., 2019).

Con base en lo anterior, es posible que en la educación presencial se estén agrupando estudiantes con mayores puntajes (Aktas et al., 2017; Cvecic et al., 2019; Coric y Aleksic-Maslac, 2010; Deng y Ye, 2018; Derbyshire et al., 2014; Ferreira y Cardoso, 2010; Ganapathy, 2015; Gibbons y Vignoles, 2012, Hamnett y Butler, 2011; Helbig et al., 2017; Ling, 2017; Lokuge, 2010; Ma et al., 2017; Smith et al., 2002; Verdis et al., 2019). En la educación a distancia dicho logro puede estar más asociado a oportunidades del acceso al estudio de estudiantes procedentes de comunidades tradicionalmente con menos acceso a la educación superior (en pobreza, de zonas rurales dispersas, indígenas o afrocolombianos, por ejemplo) donde esta opción es su única oportunidad de acceso a este tipo de educación (Aktas et al., 2017; Coric y Aleksic-Maslac, 2010; Ferreira y Cardoso, 2010; Gibbons y Vignoles, 2012; Hamnett y Butler, 2011; Helbig et al., 2017; Lokuge, 2010). Esta podría ser una explicación del cambio de distribución geolocalizada de los logros de los estudiantes de presencial y de distancia tradicional.

Los anteriores hallazgos deben tomarse con prudencia: existen diferencias importantes en el número de estudiantes en educación presencial (85.9\%), a distancia tradicional (12.7\%) y a distancia virtual (1.4\%), que podría explicar también el hecho de que el coeficiente de autocorrelación I de Moran no arrojó resultados estadísticamente significativos, por la presencia de pocos estudiantes en varios espacios geográficos analizados (Clark, 1979). Resulta pertinente realizar nuevos análisis geoespaciales (con la distancia como variable en un modelo inferencial lineal de factores con peso en el desempeño del estudiante) para examinar su potencia (Castro et al., 2018). Esta confirmación puede llevarnos a plantear si se mantiene la tendencia de concentración regional de los logros de estudiantes universitarios; o si puede tener lugar una tendencia a la ruptura de tal concentración (Arias-Velandia et al., 2018; Rincón-Báez et al., 2018; Rodríguez y López, 2016). 
Por limitaciones de comparabilidad de datos se trabajó con las pruebas de la evaluación ICFES-Saber Pro de 2012, 2013 y 2014, dentro del área de Ciencias Económicas. En la actualidad, el SNIES (2017) reporta que en la metodología virtual hay 391 programas activos de los cuales 156 son de nivel de formación de título universitario y se están ofreciendo en 14 instituciones de educación superior en Colombia, lo cual hace pensar que este panorama puede estar tendiendo a cambios o que dichos cambios pueden estar comenzando a reflejarse en los resultados recientes de evaluaciones externas del logro educativo (AriasVelandia et al., 2018; Cardona-Román et al., 2018; Rincón-Báez et al., 2018; Rodríguez y López, 2016).

\section{Referencias}

Aktas, S., Kumtepe, E., Kantar, Y., Ulukan, I., Aydin, S., Aksoy, T. y Er, F. (2017). improving gender equality in higher education in Turkey. Applied Spatial Analysis and Policy, 12(1), 1-23.

http://dx.doi.org/10.1007/s12061-017-9235-5

Arias-Velandia, N., Guarnizo-Mosquera, J., Ortiz-Romero, D., Gómez-Villareal, E. y Rojas-Benavides, L. (2018). Comienzo de la educación superior virtual en Colombia: entre la concentración geográfica de respuestas institucionales y el cierre de brechas entre zonas rurales y urbanas. En N. Arias-Velandia (Ed.), Aportes a la investigación sobre educación superior virtual desde América Latina. Comunicación, redes, aprendizaje y desarrollo institucional y social (pp. 55-72). Politécnico Grancolombiano.

https://www.poli.edu.co/sites/default/files/soportes-aportes.pdf

Arias-Velandia, N., Rincón-Báez, W., Becerra, G., Mejía, A., Salas, R. y Fajardo, H. (2018). Retos a los programas de Administración. Una Mirada al Saber. Resultados de la evaluación externa (2012-2016). Asociación Colombiana de Facultades de Administración. http://alejandria.poligran.edu.co:80/handle/10823/1192

Bravo, M., Salvo, S., Mieres, M., Mansilla, J. y Hederich, C. (2017). Perfiles de desempeño académico: la importancia de las expectativas familiares. Perfiles Latinoamericanos, 25(50), 361-386.

http://dx.doi.org/10.18504/pl2550-016-2017

Cardona-Román, D., Sánchez-Torres, J. y Acosta-Márquez, C. (2018). Panorama de la educación virtual en instituciones de educación superior en Colombia. En N. Arias-Velandia (Ed.), Aportes a la investigación sobre Educación superior virtual desde América Latina. Comunicación, redes, aprendizaje y desarrollo institucional y social (pp. 25-54). Publicaciones Politécnico Grancolombiano.

https://www.poli.edu.co/sites/default/files/soportes-aportes.pdf

Castro, M., Ruiz, J. y Guzmán, F. (2018). Cruce de las pruebas nacionales Saber 11 y Saber Pro en Antioquia, Colombia: una aproximación desde la regresión geográficamente ponderada (GWR). Revista Colombiana de Educación, (74), 63-79. https://doi.org/10.17227/rce.num74-6898

Celis, M. T., Jiménez, O. A. y Jaramillo, J. F. (2012). ¿Cuál es la brecha de la calidad educativa en Colombia en la educación media y en la superior? En Estudios sobre calidad de la educación en Colombia (pp. 67-98). ICFES.

https://www.icfes.gov.co/documents/20143/232527/Estudios+sobre+calidad+de+la+educacion+en+Colo mbia+2012.pdf

Centro Nacional de Consultoría. (2017). Evaluación de resultados del modelo de educación superior virtual del Politécnico Grancolombiano. Documentos internos de trabajo de la Institución Universitaria Politécnico Grancolombiano, contratados en el marco de este proyecto al CNC.

Clark, I. (1979). Practical geostatistics (Vol. 3). Applied Science Publishers.

Colmenares, G. (1987). La formación de la economía colonial (1550-1740). En J. A. Ocampo (Comp. y Ed.), Historia Económica de Colombia (pp. 13-47). Siglo XXI. 
Coric, M. y Aleksic-Maslac, K. (2010). E-learning potential in education sector of developing countries: Relationship marketing tool and cost cutting tool in times of financial downturn. Actas de la International Conference on Society and Information Technologies (ICSIT 2010) (pp. 7-11). Orlando, Florida.

Cortés, M. I., Norman, E. E. y Ortiz, D. A. (2019). Caracterización de proyectos de investigación formativa de programas virtuales del Politécnico Grancolombiano. Revista Electrónica de Investigación Educativa, 21, e19, 1-11. https://doi.org/10.24320/redie.2019.21.e19.1992

Cvecic, I., Sokolic, D. y Mrak, M. (2019). Higer education and economic prosperity at regional level. Revista Portuguesa de Estudos Regionais, (50), 9-25. http://www.apdr.pt/siteRPER/numeros/RPER50/50.1.pdf

Decreto 1295. (20 de abril de 2010). Por el cual se reglamenta el registro calificado de que trata la Ley 1188 de 2008 y la oferta y desarrollo de programas académicos de educación superior. Desarrollo de programas académicos de educación superior. Ministerio de Educación de Colombia.

Decreto 3963. (14 de Octubre de 2009). Ministerio de Educación Nacional. Decreto 3963 de 2009. Por el cual se reglamenta el Examen de Estado de Calidad de la Educación Superior. Ministerio de educación de Colombia.

Deng, Q. y Ye, Z. (2018). Evaluation, source of educational growth and regional comparison of higher education productivity in China. Educational Sciences: Theory and Practice, 18(5).

https://doi.org/10.12738/estp.2018.5.042

Departamento Administrativo Nacional de Estadística (2019). Codificación de la División Político Administrativa de Colombia. https://www.dane.gov.co/index.php/sistema-estadistico-nacional-sen/normasy-estandares/nomenclaturas-y-clasificaciones/nomenclaturas/codificacion-de-la-division-politicaadministrativa-de-colombia-divipola

Derbyshire, H., Rees, E., Gay, S y McKinley, R. (2014). Undergraduate teaching in UK general practice: A geographical snapshot. British Journal of General Practice, 64(623), e336-e345.

https://doi.org/10.3399/biap14X680113

El Espectador. (23 de marzo de 2014). Estas son las carreras universitarias más solicitadas por empresas en Colombia. https://www.elespectador.com/noticias/educacion/estas-son-carreras-universitarias-mas-solici tadas-empre-articulo-482544

Ferreira, S. y Cardoso, E. (2010). E-learning as an educational strategy on islands with low population density: Case study in the autonomous region of Azores. International Journal of Emerging Technologies in Learning, 5(1), 39-43. https://online-journals.org/index.php/i-jet/article/view/1063

Fujita, M., Krugman, P. R. y Venables, A. J. (2001). The spatial economy: Cities, regions, and international trade. MIT Press.

Ganapathy, K. (2015). Distribution of neurologists and neurosurgeons in India and its relevance to the adoption of telemedicine. Neurology India, 63(2), 142-154. https://doi.org/10.4103/0028-3886.156274

Gibbons, S. y Vignoles, A. (2012). Geography, choice and participation in higher education in England. Regional Science and Urban Economics, 42(1-2), 98-113.

https://doi.org/10.1016/j.regsciurbeco.2011.07.004

Guarín, A., Londoño, S., Medina, C., Parra, J., Posso, C. y Vélez, C. E. (2016). Estimating the effect of attending a public versus a private university in Colombia on academic achievement. Borradores de Economía, (968), 1-50. https://www.banrep.gov.co/en/borrador-968 
Haley, A. (2017). Defining geographical mobility: Perspectives from higher education. Geoforum, 83, 50-59. https://doi.org/10.1016/j.geoforum.2017.04.013

Hamnett, C. y Butler, T. (2011). 'Geography matters': The role distance plays in reproducing educational inequality in East London. Transactions of the Institute of British Geographers, 36(4), 479-500. https://doi.org/10.1111/j.1475-5661.2011.00444.x

Hanewicz, C. (2009). Identifying student retention patterns using GIS technology. Portland International Conference on Management of Engineering \& Technology (PICMET, pp. 2231-2239). Portland, OR., EUA. https://doi.org/10.1109/PICMET.2009.5261844

Helbig, M., Jähnen, S. y Marczuk, A. (2017). Does place of residence matter? How the geographical proximity of universities affects enrollment in higher education in Germany. Zeitschrift fur Soziologie, 46(1), 55-70. http://dx.doi.org/10.1515/zfsoz-2017-1004

Hernández, R., Fernández, C. y Baptista, M. P. (2014). Metodología de la investigación (6a. ed.). McGraw-Hill Education.

Instituto Colombiano para la Evaluación de la Educación. (2019). Bases de datos Saber Pro. https://www.icfes.gov.co/investigadores-y-estudiantes-posgrado/acceso-a-bases-de-datos

Lago, M. T., Vera, M. y Flood, C. (2004). Educación superior virtual en Argentina: un relevamiento necesario. En La educación superior virtual en América Latina y el Caribe (pp. 61-91). UNESCO-ANUIES.

Ling, M. (2017). Returning to no home: Educational remigration and displacement in rural China. Anthropological Quarterly, 90(3), 715-742. https://doi.org/10.1353/anq.2017.0041

Lokuge, K. (2010). Embracing e-learning: Higher education institutions in Sri Lanka. International Journal of Learning, 16(12), 103-110.

https://pdfs.semanticscholar.org/e324/fa179a28563160eb7ea73ada23cb6257a985.pdf

Luna, E., Ponce, S., Cordero, G. y Cisneros-Cohernour, E. (2018). Marco para evaluar las condiciones institucionales de la enseñanza en línea. Revista Electrónica de Investigación Educativa, 20(2), 1-14.

https://doi.org/10.24320/redie.2018.20.2.2072

Luo, H., Robinson, A. y Detwiler, J. (2014). Effect of geographic distance on distance education: An empirical study. Journal of Educators Online, 11(3).

https://www.thejeo.com/archive/2014 11 3/luo robinson detwiler

Ma, K., Kang, E. y Kwon, O. (2017). Migration behavior of students and graduates under prevailing regional dualism: the case of South Korea. Annals of Regional Science, 58(1), 209-233.

https://doi.org/10.1007/s00168-016-0799-9

Massey, D. y Denton, N. (1988). The dimensions of residential segregation. Social Forces, 67(2), 281-315. https://academic.oup.com/sf/article/67/2/281/2231999

Ministerio de Educación Nacional. (2014). Observación Laboral para la Educación.

http://www.mineducacion.gov.co/1621/w3-article-156290.html

Ministerio de Educación Nacional. (2020). Sistema Nacional de Información de la Educación Superior, SNIES. https://snies.mineducacion.gov.co/portal/

Montoya, D. (noviembre de 2013). Contribución de la educación superior a la equidad de resultados en Colombia. En IV Seminario internacional sobre investigación en calidad de la educación, ICFES. Bogotá, D.C. https://n9.cl/rudl 
Mora, A. (2015). La política pública de educación superior en Colombia: ¿más presupuesto, equidad y autonomía para el sistema. En C. Isaza (Ed.), Seguimiento y análisis de políticas públicas en Colombia (pp. 119134). Universidad Externado de Colombia.

Mora, A. (2016). La seudorrevolución educativa. Desigualdades, capitalismo y control de la educación superior en Colombia. Pontificia Universidad Javeriana.

Mora, A. y Múnera, L. (2019). "Ser pilo no paga": privatización, desigualdad y desfinanciamiento de la universidad pública en Colombia. Ciencia Política, 14(27), 115-142.

https://doi.org/10.15446/cp.v14n27.73369

Moran, P. (1948). The interpretation of statistical maps. Journal of the Royal Statistical Society B, 10(2), $243-$ 251. https://doi.org/10.1111/i.2517-6161.1948.tb00012.x

Padilla, A. (2004). Diagnóstico de la educación superior virtual en Bolivia. En J. A. Silvio, C. Rama y M. Lago, La educación superior virtual en América Latina y el Caribe (pp. 85-112). UNESCO-ANUIES.

The R Development Core Team. (2008). R: A language and environment for statistical computing. http://softlibre.unizar.es/manuales/aplicaciones/r/fullrefman.pdf

Restrepo, B. (2005). Consideraciones sobre el aseguramiento de la calidad en educación virtual. Consejo Nacional de Acreditación.

Rincón-Báez, W. y Arias-Velandia, N. (2017). Factores asociados al logro educativo en estudiantes en metodologías a distancia y presencial de ciencias económicas, administrativas y afines en Colombia. XXII Congreso Internacional de Contaduría, Administración e Informática-UNAM (pp. 1-18).

http://premio.investiga.fca.unam.mx/docs/XXII/4.1.pdf

Rincón-Báez, W., Arias-Velandia, N., Becerra-Plaza, G. y Durán, E. (2018). Inteligencia de datos en la formación en administración y negocios en Colombia. ASCOLFA / Institución Universitaria Politécnico Grancolombiano.

Rivera, K. (2004). La educación superior a distancia en Centroamérica. En J. A. Silvio, C. Rama, y M. Lago, La educación superior virtual en América Latina y el Caribe (pp. 145-164). UNESCO/Unión de Universidades de América Latina.

Rodríguez, G., Gómez, V. y Ariza, M. (2014). Calidad en educación superior a distancia y virtual: un análisis de desempeño académico en Colombia. Investigación y Desarrollo, 22(1), 58-99.

http://dx.doi.org/10.14482/indes.22.1.6079

Rodríguez, R. y López, D. (2016). El valor agregado de la educación superior en la formación en segunda lengua en Colombia. Civilizar. Ciencias Sociales y Humanas, 16(30), 119-135.

https://doi.org/10.22518/16578953.538

Romero, J. L. (1969/2001). La ciudad latinoamericana: continuidad europea y desarrollo autónomo. En J. L. Romero (2001). En Situaciones e ideologías en América Latina (pp. 227-234). Universidad de Antioquia.

Schabenberger, O. y Gotway, C. A. (2017). Statistical methods for spatial data analysis. Chapman and Hall/CRC.

Smith, B., Spinelli, J. y Zhoi, Y. (2002). Geographic patterns of student enrollment in Ohio's state-assisted universities. Ohio Journal of Science, 102(3), 34-39. http://hdl.handle.net/1811/23925

UNESCO. (2010). Comunicado. Conferencia Mundial de Educación Superior 2009: las nuevas dinámicas de la educación superior y de la investigación para el cambio social y el desarrollo. París.

https://unesdoc.unesco.org/ark:/48223/pf0000183277 spa 
Verdis, A., Kalogeropoulos, K. y Chalkias, C. (2019). Regional disparities in access to higher education in Greece. Research in Comparative and International Education, 14(2), 318-335.

https://doi.org/10.1177/1745499919846186

Vianney, J., Torres, P. y Farias, E. (2004). La educación superior a distancia en Brasil. En J. A. Silvio, C. Rama, y M. Lago, La educación superior virtual en América Latina y el Caribe (pp. 113-144). UNESCO/ Unión de Universidades de América Latina. 